\section{A new direction for directed mutation?}

\author{
Zhongge Zhang, Ming Ren Yen, \\ Milton H. Saier Jr. \\ Division of Biological Sciences, University \\ of California, San Diego, La Jolla, CA, \\ USA
}

\section{Abstract}

Directed mutation is a controversial process that allows mutations to occur at higher frequencies when they are beneficial. Here we review evidence for transposon-mediated directed mutation. crp deletion mutants (Glp $\left.{ }^{-}\right)$ of Escherichia coli (E. coli) mutate specifically to glycerol utilization $\left(\mathrm{Glp}^{+}\right)$at rates that are enhanced by glycerol or the loss of the glycerol repressor (GlpR), and depressed by glucose or $g l p R$ overexpression. Of the four tandem GlpRbinding sites (01-04), 04 specifically controls glpFK expression while 01 controls mutation rate. Mutation is due to insertion of the IS5 transposon into a specific site upstream of the glpFK promoter. Mutational control by GlpR is independent of the selection and assay procedures, and IS5 insertion into other gene activation sites is unaffected by the presence of glycerol or the loss of GlpR. The results establish an example of transposon-mediated directed mutation, identify the protein responsible for its regulation, and define essential features of the mechanism involved. We discuss this phenomenon from an evolutionary standpoint and provide examples of analogous switch mechanisms that may or may not be directed.

\section{Introduction}

Sixty-nine years ago, Luria and Delbrück reported that bacterial mutations from virus sensitivity to resistance arose randomly. 1 They generalized their results, concluding that genetic mutations occur in the absence of and independently of selection. It has since become a basic principle of genetics that the likelihood of a particular mutation occurs independently of its phenotypic consequences. ${ }^{1}$ The concept of directed mutation, enunciated by John Cairns and his coworkers and defined as genetic change that is specifically induced by the stress conditions that the mutation relieves, ${ }^{2}$ challenges this principle. ${ }^{3-5}$ The topic of directed mutation is controversial, and its existence, as defined above, has been altogether questioned. $6-8$
Studies of the lactose (lac) and leucine (leu) operons of $E$. coli

The most studied potential example of directed mutation involves the Escherichia coli (E. coli) lactose (lac) operon frameshift reversion system, in which cells carrying a lac +1 frameshift allele on an F' episome mutate to $\mathrm{Lac}^{+}$at a rate that is elevated by lactose during starvation. ${ }^{9}$ A subsequent genome-wide analysis showed that such $\mathrm{Lac}^{+}$point mutations (i.e., -1 deletions) may not be directed, although they are adaptive, since higher frequencies of mutation also occur simultaneously in other chromosomal genes unrelated to lactose metabolism. 10 Two mechanisms were proposed to explain the increased rates of reversion to $\mathrm{Lac}^{+}$, one involving double-strand break repair mediated by error-prone DNA polymerase IV,11 the other involving amplification of the leaky lac frameshift allele, thereby amplifying the low activity of the mutant allele.7,12 The first model was supported in a follow-up study. ${ }^{13}$ Both lac allele amplification and $\mathrm{Lac}^{+}$point mutations were induced by lactose starvation and regulated by the stationary phase regulator, RpoS.14,15 These two processes have been shown to be two independent outcomes of genetic change during starvation, and both of them are adaptive processes. ${ }^{16,17}$

Amino acid biosynthetic auxotrophs of $E$. coli can be mutated to grow in the absence of the required amino acids. One such auxotroph, which contains a C-to-T point mutation at nucleotide 857 in the $l e u B$ gene (leading to a S286L change of the protein), reverts to $\mathrm{Leu}^{+}$ when the $\mathrm{Leu}^{-}$cells are incubated under leucine-limited conditions. 18 Thirty six out of $53 \mathrm{Leu}^{+}$revertants or pseudorevertants were found to harbor a single nucleotide substitution that resulted in alteration of the $286^{\text {th }}$ residue from leucine back to serine, or to valine or methionine. 18 These Leu+ reversion mutations proved to occur in response to leucine starvation. The increased rate of Leu+ mutation is believed to have resulted from increased rates of transcription elicited by increased cytoplasmic concentrations of guanosine tetraphosphate (ppGpp), ${ }^{19,20}$ an alarmone whose synthesis is increased in response to leucine starvation. ${ }^{21}$ Transcription-promoted Leu+ mutation is probably an example of adaptive mutation, and could be a potential example of directed mutation since most (but not all) of the identified mutations seemed to be directed to the leuB gene whose product is needed for the $\mathrm{Leu}^{+}$ phenotype. However the involvement of ppGpp suggests that this response may also be pleiotropic (affecting other operons under ppGpp control) rather than being operon specific.

Part of the justifiable skepticism concerning directed versus adaptive mutation of the lac system in E. coli resulted from experiments
Correspondence: Milton H. Saier Jr., Division of Biological Sciences, University of California, San Diego, La Jolla, CA 92093-0116, USA.

Tel: +1.858.534.4084 - Fax: +1.858.534-7108.

E-mail: msaier@ucsd.edu

Key words: directed mutation, adaptive evolution; transposon; insertion sequence; transposition regulation.

Acknowledgements: some material and figures were reproduced or modified from Zhang \& Saier 2009 (49) or Zhang, Z., Yen, M.R. \& Saier, M.H., Jr. 2010 (63) with permission. We thank Jeeni Criscenzo and Carl Welliver for assistance in the preparation of this manuscript. The work reviewed here was supported by NIH grants GM 64368 and GM 077402.

Received for publication: 2 December 2010.

Revision received: 19 July 2011.

Accepted for publication: 1 August 2011.

This work is licensed under a Creative Commons Attribution NonCommercial 3.0 License (CC BYNC 3.0).

(C) Copyright Z. Zhang et al., 2011

Licensee PAGEPress, Italy

Trends in Evolutionary Biology 2011; 3:e3 doi:10.4081/eb.2011.e3

that supposedly demonstrated this phenomenon ${ }^{2,9}$ but were subsequently shown to be explainable by classical genetics.8,22 Mutation rates vary with environmental conditions and genetic background (e. g., the presence of mutator genes).5,23,24 However, this does not render the mutation directed. To convincingly establish the principle of directed mutation, it is necessary to demonstrate the specificity of the phenomenon, identify the proteins involved, and characterize the mechanism responsible.

\section{Transposon hopping as a means of gene activation}

Transposons such as Insertion Sequence 5 (IS5) provide the host organism with benefits such as the opportunity for genetic changes that might, for example, relieve a stress condition or allow utilization of a nutrient not otherwise usable. These jumping genes can transpose by at least two distinct mechanisms, one involving replication, $25-27$ the other being replication independent. ${ }^{26,28}$ More than 500 transposons have been identified to date, and they are commonly found in the genomes of both prokaryotes and eukaryotes. ${ }^{29}$ They are believed to be primary causes of DNA rearrangements including chromosomal inversions and deletions, ${ }^{30-32}$ all of which could be beneficial under specific stress conditions.

The hopping of transposable elements, transposons, can activate or inactivate critical 
genes or operons when inserted into appropriate chromosomal loci.33-35 Transposon-mediated mutations occurring under stress conditions (e.g. starvation or the presence of a toxin) can be beneficial to the host organisms. For example, the products of the $E$. coli $n f s A B$ operon convert nitroaromatic compounds to toxic nitro-anion free radicals via nitroreduction. ${ }^{36}$ When $E$. coli is exposed to these compounds in agar plates, resistant colonies appear during incubation, and all such mutants arise due to insertion of IS1 or IS5.37 Activation of the normally cryptic $\beta$-glucoside $(b g l)$ catabolic operon ${ }^{38,39}$ and the ade gene, encoding an adenine deaminase in $E$. coli, 40 can be accomplished by insertion of either IS1 or IS5 near the promoter. IS5 has also been found to activate the fucose (Fuc)/propandiol (Ppd) $f u c A O$ promoter ${ }^{41}$ as well as the flagellar motility flhDC master switch promoter. 42 These studies, while not shown to be directed, provided the background for recent studies on the $E$. coli glpFK operon in which IS5-mediated activation occurs in a directed fashion by a well defined mechanism.

\section{Structure of the $E$. coli glp regulon}

The $E$. coli glp regulon consists of five operons, two of which (glpFK and glpD) are required for aerobic growth on glycerol. 43 The glpFK operon encodes the glycerol transport facilitator, GlpF, allowing rapid entry of glycero into the cell, and glycerol kinase, GlpK, converting glycerol to glycerol-3-phosphate. The $g l p D$ gene encodes the aerobic glycerol-3-P dehydrogenase that oxidizes glycerol-3-P to the glycolytic intermediate, dihydroxyacetone phosphate.43,44 Both operons are subject to negative control by the DNA-binding $g l p$ regulon repressor, GlpR, 45 which also binds glycerol-3-phosphate, the inducer of the $g l p$ regulon. The $g l p F K$ operon, but not $g l p D$, is additionally subject to positive regulation by the cyclic AMP receptor protein, Crp, complexed with cAMP.46 The glpFK regulatory region contains four GlpR binding sites, 01-04, and two Crp binding sites which overlap 02 and 03 (Figure 1). The strong Crp dependency of glpFK transcription is reflected by the fact that crp mutant cells are unable to utilize glycerol.

\section{A novel mechanism of directed mutation?}

In the absence of Crp, the $g l p F K$ promoter can be activated by IS5 when this genetic element inserts upstream of the promoter. ${ }^{47}$ High level expression of the resultant activated operon is nearly constitutive and relies on the DNA phase between the inserted IS5 and the promoter. A short region of $177 \mathrm{bps}$ (IB) at the 3 ' end of IS5, which contains an IHF binding site and A-tracts that generate a permanent bend, ${ }^{48}$ is necessary and sufficient for such activation. Moreover, IS5 or IB, when inserted into appropriate sites, can activate other Crpcontrolled promoters such as the lac promoter of $E$. coli. 47

This insertional event appears to represent a genuine example of directed mutation. Mutation is mediated by IS5 insertion at a specific site upstream of the glpFK promoter. Mutation of a crp deletion mutant to $\mathrm{Glp}^{+}$ occurs with a ten fold higher frequency when glycerol is present or GlpR is lacking, but overexpression of $g l p R$ greatly depresses the mutation rate. ${ }^{49}$ Frequencies of IS5 insertion to other sites that activate dissimilar promoters were unaffected by glycerol or the loss of GlpR. GlpR therefore seems to provide two distinct biological functions, one, recognized previously, to control gene expression by binding to the downstream operator, 04 , and the other, to control the IS5-dependent mutation rate by binding to the upstream operator, $01 .{ }^{49}$ This is the first example of transposon-mediated directed mutation where the molecular explanation, involving a DNA-binding protein, has been provided. Mechanistic details will be presented below.

\section{$\mathrm{GIP}^{+}$mutations in a crp genetic background}

E. coli cells that lack Crp can not utilize glycerol as the sole carbon and energy source since expression of the $g l p F K$ operon is essential for glycerol uptake and metabolism. However, when $\operatorname{crp}$ cells are incubated on solid glycerol minimal medium, Glp ${ }^{+}$colonies appear after prolonged incubation. ${ }^{49}$ Dozens of crp Glp ${ }^{+}$ mutant colonies from different plates and arising on different days were purified and tested for growth on glycerol in defined liquid medi- um. All such mutants grew equally well. The growth curve for one such mutant is shown in Figure 2A. The lag phase for the $c r p$ Glp $^{+}$strain was shorter, and its growth rate was slightly greater than that of wild type $(w t) E$. coli. Using Biolog plates, these two strains (parental crp Glp- and crp Glp+ cells) were tested for oxidation of other biological carbon, nitrogen, phosphorous and sulfur sources, but no obvious differences were observed. ${ }^{49}$ The mutation that enables $c r p$ cells to utilize glycerol evidently did not affect other phenotypes.

The relative frequencies of $\mathrm{Glp}^{+}$mutation were determined with glycerol, sorbitol or glucose as the sole carbon source on minimal agar plates. On glycerol plates, colonies appeared first after 3 days although $w t$ and $c r p$ $\mathrm{Glp}^{+}$cells formed visible colonies in $<2$ days. New colonies continued to appear at increasing frequencies thereafter. When the same $c r p$ cells were plated as before, but variable numbers of the cells from a crp $\mathrm{Glp}^{+}$strain were included with the cells before plating, colonies appeared from the $\mathrm{crp} \mathrm{Glp}^{+}$cells within two days, and new Glp ${ }^{+}$mutants arose at the same frequency as before (Figure 2B). This experiment was repeated many times using independently isolated $c r p \mathrm{Glp}^{+}$mutant strains, and they all behaved similarly. Thus, the Glp ${ }^{+}$ mutants arising from crp cells on these glycerol minimal medium plates were not present in the cell culture before plating, and the potential presence of a growth inhibitor could not account for the results. The ratio of $\mathrm{Glp}^{+}$ colonies (independent mutations that occur after plating) to the total population increased with time. When sorbitol (another sugar crp cells can not utilize) replaced glycerol, the frequency of appearance of $\mathrm{Glp}^{+}$mutations was much lower than that on glycerol plates but

$$
\begin{aligned}
& \overbrace{\text { aatgatćtaaćaaacatgcatcatgtacaatcagatggaataa } \overline{\text { atggcgc }}}^{-122}
\end{aligned}
$$

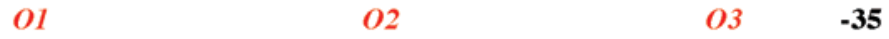

$$
\begin{aligned}
& \overline{\text { taacgctcatt }} \overline{t \text { tatgacgaggcacacacatt }} t \overline{\text { taagttcgatatttctcg }} \\
& 0 4 \longdiv { - 1 0 } { } ^ { \operatorname { C r p ~ I } + 1 } \quad \text { Crp II } \\
& \overline{\mathrm{t} t} \overline{\mathrm{ttgctcgttaacgataag} t} \mathrm{ttacag} \text { catgcctacaagcatcgtggaggt } \\
& \text { ccgtgactttcacgcatacaacaaacattaactcttcaggatccgattatg }
\end{aligned}
$$

Figure 1. The $E$. coli $g l p F K$ promoter region. The transcriptional initiation site (+1), the -10 and -35 hexamers that comprise the promoter, the ribosome binding site (RBS) and the start codon for $g l p F$ translation are shaded. The GlpR binding sites (O1-O4; lines above the sequence) and $\mathrm{Crp}$ binding sites (CrpI and $\mathrm{CrpII}$; lines under the sequence) are also shown. The location of the IS5 element $37 \mathrm{bp}$ upstream of $\mathrm{O1}$ in $\mathrm{crp} \mathrm{Glp}^{+}$cells is indicated by the vertical white arrow below the horizontal green arrow representing IS5. This arrow shows the orientation of IS5 with the 3' end of its transposase gene linked to the downstream promoter. The 4-nucleotide IS5 target sequence (CTAA) at -126 to -122 is shaded. Reproduced from reference 49. 
substantially higher than that on glucose plates where mutation rates were negligible.

\section{IS5 upstream of PgIpFK in the crp \\ $\mathrm{Glp}^{+}$mutants}

The $g l p F K$ regulatory region from 116 out of 116 independently isolated Glp+ colonies contained a $\sim 1.1 \mathrm{~kb}$ insert that proved to be IS5, located between the $127^{\text {th }}$ nucleotide and the 126th nucleotide upstream of the glpFK transcriptional start site. It was always orientated with the 3 ' end proximally upstream of the promoter (Figure 1). The four base IS5 recognition sequence, CTAA, was repeated immediately adjacent to IS5 as expected. 35,49 No other mutation in addition to the IS5 insertion could be detected, and other DNA elements inserted at the same site did not activate glpFK-expression. Furthermore, insertion of an 85bp fragment either within or downstream of the CTAA IS5 target site essentially abolished $(<2 \%$ frequency) appearances of $\mathrm{Glp}^{+}$mutants, and insertion of the same element upstream of this tetranucleotide target sequence reduced the insertion frequency to about 50\%. These observations showed that (i) the CTAA insertion site upstream of $P g l p F K$ is required for IS5 hopping to this region, (ii) an appropriate location of the CTAA element upstream of PglpFK is essential for $g l p F K$ operon activation, and (iii) the adjacent sequence upstream of the CTAA target site is important to maximize the IS5 insertion rate.

\section{Dependence of the $\mathrm{Glp}^{+}$mutation rate on the glycerol repressor, GlpR}

Glycerol is phosphorylated by GlpK to glycerol-3-phosphate which binds to and releases the GlpR repressor from its operators. 43,46 When GlpR dissociates from its operators, a conformational change might be transmitted through the DNA, promoting insertion of IS5 at the CTAA site upstream of PglpFK.

To test this possibility, the $g l p R$ gene was deleted, and the frequencies of appearance of $\mathrm{Glp}^{+}$mutations in the $\mathrm{crp}$ single mutant and the $\operatorname{crp} g l p R$ double mutant were measured in the absence and presence of glycerol. When the $c r p$ and $c r p g l p R$ strains were plated without glycerol, individual Glp ${ }^{+}$colonies from the crp glpR double mutant formed on the plates with a 10-fold higher frequency than from the crp mutant. In the presence of glycerol, similar mutation frequencies were observed for the $c r p$ and $c r p$ glpR strains, and these rates were similar to those observed for the $c r p$ glpR double mutant in the absence of glycerol. These experiments demonstrated that deletion of $g l p R$ is equivalent to inclusion of excess glycerol in the growth medium. GlpR therefore mediates the response to glycerol.49

To further demonstrate that GlpR binding to the DNA inhibits the appearance of Glp+ muta- tions, the $g l p R$ gene was cloned into a vector and expressed under the control of an inducible promoter. When cells expressing the $g l p R$ gene at high levels were quantitated for the appearance of $\mathrm{Glp}^{+}$mutants, the frequencies of these mutations decreased below the background rate observed for the $c r p$ strain. It thus became clear that GlpR controls transposition of IS5 to the site upstream of the $g l p F K$ promoter. But what about other sites on the $E$. coli chromosome? Examination of three other operons known to be activated by IS5 insertion, the fucAO,41 flhDC 42 and $b g l G F B$ operons, 38,39 revealed that neither the presence of glycerol nor the loss of GlpR influenced the IS5 hopping rates to these sites. It was therefore concluded that GlpR is site specific, only influencing IS5 mediated mutation rate by transposition of IS5 to the activating site upstream of the $g l p F K$ promoter.

\section{GlpR operators differentially con- trol glpFK expression and Glp ${ }^{+}$ mutation rate}

DNA footprinting identified four GlpR binding sites, $01-04$, in the upstream glpFK operon regulatory region 45 (Figure 1). The far
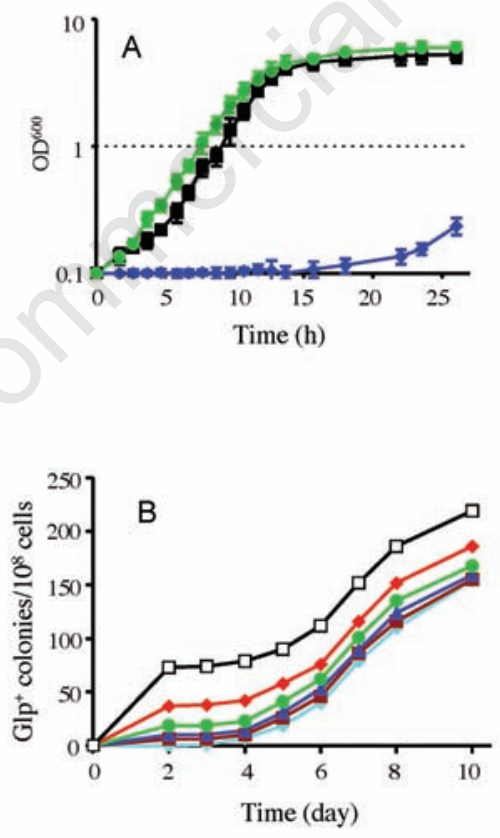

Figure 2. The appearance of $\mathrm{Glp}^{+}$mutations in a crp genetic background. (A)

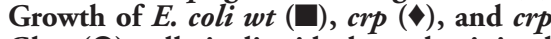
$\mathrm{Glp}^{+}(\mathbf{O})$ cells in liquid glycerol minimal medium. (B) Growth in the same medium, where the crp mutant cells were plated together with varying numbers of cells from a $\operatorname{crp}$ Glp $^{+}$strain: $(\square, 72 ; \emptyset, 38$; $\bullet$, $19 ; \Delta, 10 ; \square, 5$; and $\downarrow, 0)$. The $\operatorname{crp} \mathrm{Glp}^{+}$ cells were mixed with crp cells $\left(10^{8}\right)$ and then applied onto M9 glycerol agar plates. Six independently isolated $\mathrm{Glp}^{+}$mutant strains behaved the same. Reproduced from reference 49. upstream site $(01)$ and the far downstream site (04) were mutated, and the effects on glpFK expression and mutation rate were compared. ${ }^{49}$ For glpFK expression measurements, a lac $Z$ reporter gene fusion construct was used (Figure 3A), and for meaurement of the frequency of mutation to $\mathrm{Glp}^{+}$, a standard cell counting procedure was used (Figure 3B). Mutation of 04 increased $g l p F K$ operon expression about 5 -fold although mutation of $\mathrm{Ol}$ was almost without effect (Figure 3A). In contrast, loss of 01 yielded a sevenfold increase in mutation frequency although loss of 04 had only a 2 -fold effect (Figure 3B). Thus, while 01 primarily controls mutation rate, 04 primarily controls operon expression. Mutation rate is therefore not a function of $g l p F K$ expression level, and GlpR regulates expression and mutation rate independently.
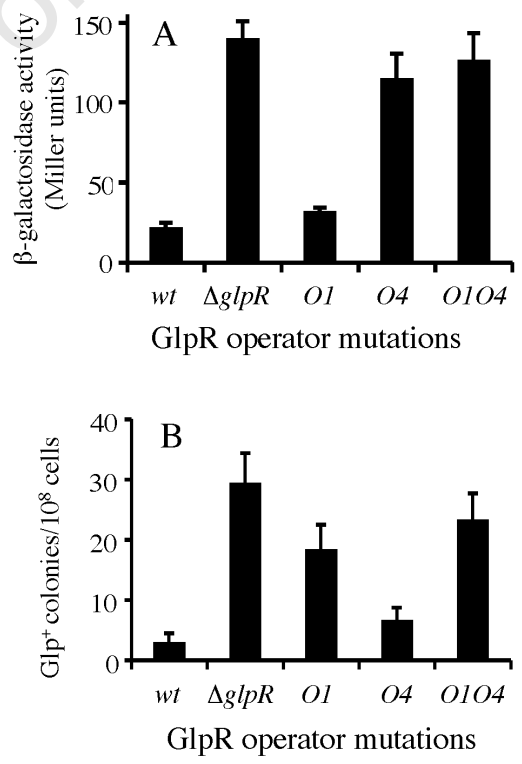

Figure 3. Effects of the losses of GlpR and its binding site operators, $\mathrm{Ol}$ and $\mathrm{O} 4$; $\triangle g l p R$, deletion of the $g l p R$ gene; on (A) glpFK operon expression levels and (B) IS5-mediated mutation rates when grown in liquid LB medium. The frequencies of $\mathrm{Glp}^{+}$mutations relative to the total cell populations were plotted versus time, and the mutation rates were determined from the slopes of the curves. The graphs show the effects of mutations in $g l_{p} R$ and its operators, $O 1$ and $O 4$ (Figure 1), on (A) PglpFK activity in $\operatorname{crp}$ cells grown in LB

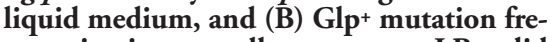
quencies in crp cells grown on LB solid medium. The bars represent standard deviation values for three independently conducted experiments. wt: control with no mutation in either 01 or 04 ; O1: only operator 1 was mutated; $O 4$ : only operator 4 was mutated; 0104 : both operators were mutated. Reproduced from reference 49. 
IS5 insertion rates using the chloramphenicol $(\mathrm{Cm})$ resistance gene (cat) for selection

To confirm the effects of glycerol and GlpR on IS5 insertion upstream of the $g l p F K$ promoter and to show that control of mutation rate by GlpR is independent of the Glp+ phenotype, the chromosomal glpFK operon was replaced with a chloramphenicol resistance (cat) structural gene so that cat was expressed solely from the glpFK promoter (i.e., PglpFK-cat at the glpFK locus). crp and crp glpR cells proved to be sensitive to $\mathrm{Cm}$ at $<25 \mu \mathrm{g} / \mathrm{mL}$, but IS5 insertion as reported above rendered these cells resistant to $\mathrm{Cm}$ at $>50 \mu \mathrm{g} / \mathrm{mL}$. Using this chromosomal PglpFK-cat construct, IS5 insertion assays were performed by incubating cells on LB agar plates with $50 \mu \mathrm{g} \mathrm{Cm}$ per $\mathrm{mL}$. Cm resistant $(\mathrm{Cm} r)$ colonies arose on both $c r p$ and $c r p g l p R$ plates, but the rate of appearance of $\mathrm{Cm}^{r}$ colonies relative to the total populations was about 20 times higher for the $c r p g l p R$ cells than for the $c r p$ cells (Figure 4). Furthermore when $g l p R$ was overexpressed, the rate at which $\mathrm{Cm}^{\mathrm{r}}$ colonies arose decreased dramatically compared to the same cells carrying the empty plasmid. ${ }^{49}$ Sequencing analysis showed that 20 out of 20 independently isolated $\mathrm{Cm}^{\mathrm{r}}$ mutants from either $c r p$ cells or $c r p$ glpR cells carried IS5 in the usual location and orientation. These results support the conclusions that (i) GlpR represses the appearance of Glp ${ }^{+}$ mutations in the absence of glycerol; (ii) IS5 insertion upstream of PglpFK is the sole cause of the Glp+ phenotype, and (iii) regulation of mutation rate still occurs when a phenotype unrelated to glycerol metabolism is used to measure mutation rate.

\section{RecA independence of $\mathrm{Glp}^{+}$muta- tion}

The increased mutation rate in response to the loss of GlpR binding might have resulted from increased gene dosage accompanying homologous recombination-dependent partial chromosomal duplications.22,50,51 Such duplications are RecA-dependent. ${ }^{51}$ The dependency of $\mathrm{Glp}^{+}$mutation on RecA was therefore examined. When cells were incubated on LB agar plates, and individual colonies were examined for $\mathrm{Glp}^{+}$and total populations, only a $15 \%$ apparent decrease in mutation frequency was observed in $\mathrm{crp}$ recA double mutant cells compared to $c r p$ cells. Similarly, when cells were incubated on minimal glycerol agar plates, introduction of the recA mutation decreased the $\mathrm{Glp}^{+}$mutation frequency by only $15 \%$. It is therefore clear that the effect of glycerol or the binding of GlpR to its $g l p F K$ operators is not dependent on $\operatorname{RecA}$ and therefore is not dependent on homologous recombination for partial chromosomal duplication.
The mechanism of IS5-mediated activation of the glpFK promoter

The results summarized above lead to the conclusion that IS5 insertion into a single chromosomal locus in front of the glpFK promoter occurs under starvation conditions with high frequency when glycerol is present and glucose is absent. These are the same conditions that allow these IS5 insertional mutations to be beneficial by relieving the starvation stress. However, the experiments described above do not address the mechanism by which IS5 activates the $g l p F K$ promoter.

The promoter activation effect of IS5 proved to be solely due to a short (177bp) region at the 3 ' end of IS5. ${ }^{47}$ This region is both necessary and sufficient for full promoter activation. It harbors a permanent bend, due to the presence of appropriately spaced A-tracts52-54 and an overlapping binding site specific for the genome shaping histone-like protein, Integration Host Factor (IHF). 55 Both of these elements proved to be required for full promoter activation. ${ }^{47}$ When each was eliminated by mutation, about $50 \%$ of the activation was lost, and their effects were additive. When both were lost, no activation was observed. ${ }^{47}$ In support of the conclusion that DNA bending provides the basis for activation by both the Atracts and IHF binding to its site within the 177bp sequence at the end of IS5, phasing of the DNA proved to be important. 47 Thus in BDNA a helical turn is about 10bp long, and when a 10bp segment was inserted, there was only a slight loss of $g l p F K$ expressional activation, but when a 5bp sequence was inserted at the same location, activation was totally lost.

The mechanism thus appears to involve
DNA bending. Presumably the upstream region of the DNA interacts with the transcriptional initiation complex to activate the glpFK promoter that is normally activated by the cyclic AMP-Crp complex in wild type cells. Additionally, it was shown that the $E$. coli lactose (lac) operon could be activated by the 177bp IB fragment in a crp genetic background. These observations suggest that this newly demonstrated activation mechanism could be applicable to many catabolite controlled operons in a variety of bacteria. It would

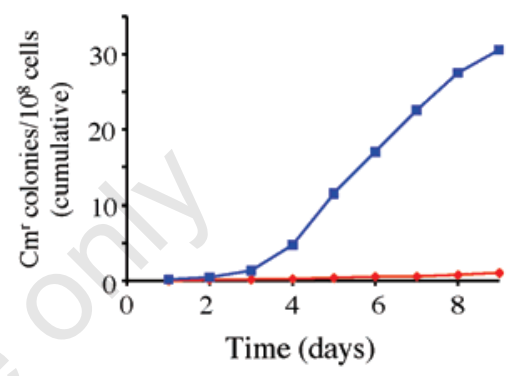

Figure 4. Effects of GlpR on the frequencies of IS5 insertion upstream of PolpFK when this promoter drives expression of a cat [chlorophenicol resistance $(\mathrm{Cm})]$ gene. The graph reveals the effect of the loss of GlpR on the frequency of IS5 insertion upstream of $P g l p F K$ in $\operatorname{crp}(\downarrow)$ and $\operatorname{crp} g l p R$ (-) genetic backgrounds. GlpR overproduction depressed the rate of mutation below background levels. The results show that control of mutation rate by the binding of GlpR to the DNA is independent of the selection/detection assay. Reproduced from reference 49 . fic $A O+$ cattagctacctctctctgattcaaaacagggcaataatgttgttccttcacactattgaattagccgtttaattacccacc -351 atcttcttcctgattaacaagaaagaaattcacaagcttatatttgtgacctggttcaactaatcacagtaaataactgcaagttctctt -260 tttataaccccattaaaatgaccgctcttaaaaatttatcaaaacggtcattttctattcctccaagcceggaatgaccgtttcg -170 gcacaaacaattaatacggtcatctgatttgtgtttttatgatttattttctgaaacgggcatgaaatttcgattattaaagtgatggtag -78

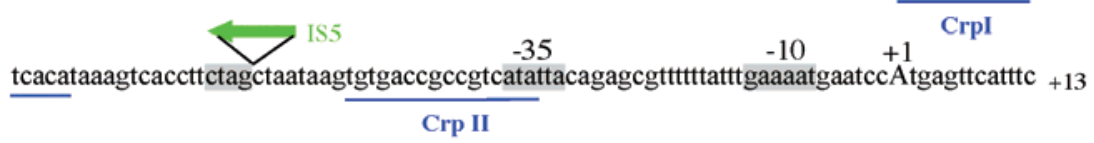

agacaggcaaatattcactgatatgaagcccgaactcgctggtttgcactttgaaaacataaccgattacgtgcttaagcttctga +101

RBS start codon

acctaagaggatgctatg + fuc $P I K$

Figure 5. The interoperonic region between the fucPIK and fucAO operons. In the fucPIK promoter region, the transcriptional initiation site $(+1)$ is capitalized. The ctag IS 5 target site, the -10 and -35 hexamers, the ribosome binding site (RBS) and the ATG start codon for fucP are shaded. The two putative Crp binding sites (CrpI and CrpII) are underlined. The green backward arrow above the sequence indicates the location of IS5 upstream of the fucPIK operon in Ppd $+/$ Fuc- cells. The arrow shows the orientation of IS 5 with the defined left end linked to the downstream fuc $A O$ promoter. In the fuc $A O$ promoter region, the putative ribosome binding site and the start codon for fuc $A$ are shaded. The promoter region for the fucAO operon is unknown. This operon has been shown to be under positive control by Crp, but the Crp binding sites have only been identified bioinformatically, not experimentally. Modified from reference 63. 
not be unexpected if other operons, not under Crp control, will prove to be subject to this IS5mediated mechanism as well. Moreover, other transposons may have similar capacities to use an analogous mechanism or a variant of it for promoter activation. Further experimentation will be required to establish or refute these possibilities.

\section{Precise excision of IS5}

Some transposable elements have been shown to excise both precisely and imprecisely, with precise excision usually occurring at much lower rates than imprecise excision.56-58 In one study, IS5 was found to be excised imprecisely, causing deletion of adjacent regions. ${ }^{59}$ Until recently, no information had been available regarding precise excision of IS5.

The fuc regulon for L-fucose (Fuc) uptake and metabolism consists of two divergent operons, fucPIK and fucAO (Figure 5). fucPIK encodes a permease (P) for fucose uptake, an isomerase (I) that converts fucose to fuculose, and a kinase $(\mathrm{K})$ that phosphorylates fuculose to fuculose-1-P; fucA encodes an aldolase that cleaves fuculose-1-P to lactaldehyde and dihydroxyacetone-P. Under aerobic conditions, lactaldehyde is further metabolized to pyruvate that subsequently enters the TCA cycle. Under anaerobic conditions, lactaldehyde is converted to L-1,2-propanediol (Ppd) by Fuc0, and Ppd then diffuses out of the cell.60 Expression of both operons relies on the cyclic AMP receptor protein, Crp, and the activator of the fuc regulon, FucR. Binding of fuculose-1-P to FucR promotes DNA binding and stimulates transcription of the two fuc operons. ${ }^{60}$

$E$. coli cells can not utilize propanediol for growth since neither fuc operon can be activated by this carbon source. However, when IS5 hops into the intergenic region between the two operons (Figure 5), the cells gain the ability to utilize propanediol. ${ }^{41,61}$ The presence of IS5 causes constitutive expression of the fucAO operon but prevents expression of the fucPIK operon, possibly because it relocates a Crp binding site in the control region of this latter operon. 62

IS5 insertion into the fuc control region allows utilization of propanediol $\left(\mathrm{Ppd}^{+}\right)$, but simultaneously prevents utilization of fucose (Fuc ${ }^{-}$). ${ }^{62}$ Wild type $E$. coli cells are Fuc ${ }^{+} \mathrm{Ppd}^{-}$, but when IS5 hops into the critical site within the regulatory region between the fucAO and fucPIK operons, the former operon is activated while the latter one is silenced. This results in a dual phenotypic change, from Fuc ${ }^{+}$Ppd- to Fuc $^{-} \mathrm{Ppd}^{+}$. This provides positive selection for IS5 insertion $\left(\mathrm{Ppd}^{+}\right)$, and also for precise IS5 excision $\left(\mathrm{Fuc}^{+}\right)$.

Assuming that transposons have at least in part evolved to effect genetic change by gene activation or inactivation under stressful con- ditions, one might assume that under other conditions (such as when fucose, but not propanediol, is available as a source of carbon), it would be beneficial to precisely excise the element, restoring the original phenotype. Without a mechanism to fully reverse the mutation-induced phenotypic change, IS transposition could be just as deleterious in the long run as it could be beneficial under a specific set of environmental conditions.

Recently, precise excision of IS5 from the interoperonic region between fucPIK and fucAO has been demonstrable because of the positive selection procedures described above.63 The demonstration of precise excision, first by full phenotypic reversion, and then by DNA sequencing, established the occurrence of full reversibility of the transposition event.63 This demonstration shows that precise excision of IS5 can occur, increasing its potential usefulness for adaptive purposes.

\section{Conclusions}

Directed mutation has been defined as a genetic change that is specifically induced by the stress condition that the mutation relieves (see Introduction). Although several such potential processes have been studied, seldom have the mechanisms been determined ${ }^{3,19,20,64}$ In this review, we summarize some of the early work on directed mutation as well as recent evidence suggesting that mutations are directed to the $g l p F K$ control region, thereby allowing growth of $E$. coli crp mutants on glycerol. These mutations are specifically induced by the presence of glycerol under starvation conditions. The $g l p$ regulon-specific protein that regulates mutation frequency was identified, and the control mechanism was in large measure elucidated. The glycerol regulon repressor, GlpR, which binds to its four operators (O104 ) in front of the $g l p F K$ operon, is displaced from these sites when $\alpha$-glycerol phosphate, derived from glycerol by phosphorylation, is bound (Figure 1).46 GlpR clearly controls both glpFK expression and the Glp+ mutation frequency. 04 , which overlaps the -10 region, primarily influences gene expression, 02 and 03 , which overlap the two Crp binding sites and the -35 promoter region, presumably antagonize activation by the cyclic AMP-Crp complex in wild type cells, and 01 primarily influences IS5 hopping into the specific CTAA site, 126.5 base pairs upstream of the $g l p F K$ transcriptional start site, 37 bps upstream of $O 1$ (Figure 1). The results serve to dissociate the two functions of GlpR. The mutational inhibitory mechanism, dependent on the binding of GlpR to 01 , may be direct, involving interaction of DNA-bound GlpR with the transpososome, or indirect, involving changes in DNA conforma- tion (e.g., supercoiling, secondary structure, etc).

Adaptive $\mathrm{Lac}^{+}$mutations that arose during lactose selection appeared not to be directed specifically to the gene in which mutation relieved the stress. ${ }^{6,10}$ However, in the case of $\mathrm{Glp}^{+}$mutations, it was shown that (i) each of 116 independently isolated Glp+ mutants contains an IS5 copy, always present at the same location; (ii) only one of the five $g l p$ operons ( $g l p F K$ ) is activated; (iii) the presence of glycerol or the loss of GlpR did not affect IS5 hopping into three other gene activating sites in the genome; and (iv) there are no observable differences between $\mathrm{Glp}^{+}$mutants and the parental crp cells in the utilization of other carbon, nitrogen, sulfur and phosphate sources. 49 These facts strongly suggest that mutation to $\mathrm{Glp}^{+}$is directed specifically to the $\mathrm{glpFK}$ operon, while other IS5-mediated gene activating events do not occur at altered rates when glycerol is present or the $g l p R$ gene is deleted. These results are therefore fully compatible with the most rigorous definition of true directed mutation.

Several mechanisms have been proposed to account for increased rates of mutation in $E$. coli cells under stress conditions. 65 Two of these (a point mutagenesis mechanism and an adaptive gene amplification mechanism) have been proposed to promote lac frameshift reversion.65 The point mutagenesis mechanism includes DNA double-strand breaks and their subsequent repair, executed by error-prone DNA polymerase IV that is induced under stress conditions such as starvation. In growing cells, double-strand break repair is mediated by a high-fidelity polymerase, such as PolI.11,13 The gene amplification mechanism involves increasing the copy number of the lac +1 allele to up to 50 tandem repeats per cell. ${ }^{\text {, }}$ $12,17,66$ Each copy of the allele accounts for 1 to $2 \%$ of the wild type level of $\beta$-galactosidase. Therefore, a sufficient level of expression of the leaky allele causes the $\mathrm{Lac}^{+}$phenotype ${ }^{7,12,16}$

Another mechanism for increasing mutation rate involves the guanosine tetraphosphate (ppGpp)-mediated elevation of transcription of amino acid metabolic genes in response to nutritional stress, thereby enhancing the amount of local single-stranded DNA, which is more vulnerable to mutation. ${ }^{19,20}$ The most studied example of transcription-elicited mutation is the $\mathrm{Leu}^{+}$reversion of an $E$. coli $l e u B$ auxotroph during leucine starvation. 5 , 18,19 A fraction $(\sim 25 \%)$ of the $\mathrm{Leu}^{+}$mutants proved not to be true revertants, and they contained one or more mutations other than a nucleotide substitution in the $l e u B$ gene.18 Because this mechanism is dependent on ppGpp, it is not likely to be specific for leuB.

These reported mechanisms appear entirely different from the Glp ${ }^{+}$mutation mechanism reviewed here. In the case of $\mathrm{Glp}^{+}$mutations, 
(i) no evidence suggests an involvement of DNA double-strand breaks or Pol IV-mediated error-prone repair; (ii) homologous recombination-mediated gene amplification proved not to be responsible for the elevated mutation rate induced by the presence of glycerol or the loss of GlpR; (iii) the Glp ${ }^{+}$mutation rate is not a function of the $g l p F K$ expression level, and (iv) binding of a local protein (GlpR) to one of its four operators, 01, depresses the Glp ${ }^{+}$ mutation rates in the absence of glycerol. 49

In several cases, transposon insertion frequencies have been shown to increase in the presence of specific carbon sources.67,68 A series of host proteins have been shown to increase or decrease transposition of transposons in E. coli. $33-35,69$ Many of these proteins are either nucleoid structuring proteins such as $\mathrm{H}-\mathrm{NS}, \mathrm{HU}$, IHF and Fis ${ }^{55}$ or proteins related to DNA recombination and repair such as RecG, Dcd, and DinD.70,71. Often, increased

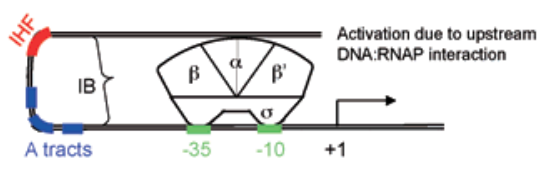

Figure 6. Model for the activation of $g l p F K$ expression by the upstream IS 5 in $E$. coli K12. IB: IS5 permanent bend/IHF binding region in the downstream part of IS5; $\alpha, \beta$, $\beta$, $\sigma$, the four dissimilar subunits of $E$. coli RNA polymerase. The -10 and -35 represent the two sigma-70 $\left(\sigma^{70}\right)$ binding sites in the promoter region to which $\sigma^{70}$ binds for open complex formation.

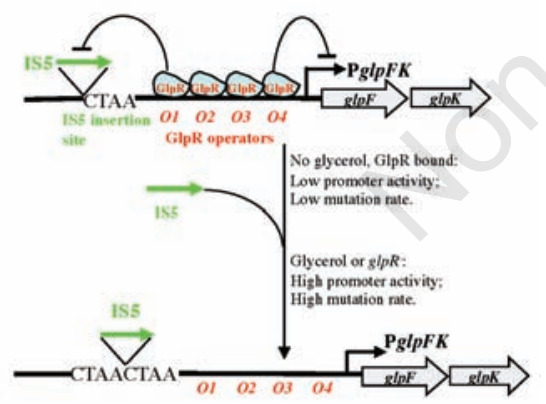

Figure 7. Schematic diagram illustrating GlpR-mediated control of (right) $g l_{p F K}$ transcription and (left) the rate of IS5 hopping (directed mutation) into the CTAA site upstream of the $g l_{p} F K$ promoter. With GlpR bound to its operators (O1-O4), transcription and IS5 hopping both occur at low rates. When GlpR is not bound to its operators, both transcriptional initiation and IS5 hopping increase about 10-fold. Binding of GlpR to operator $\mathrm{O} 1$ blocks IS5 insertion, while binding of GlpR to operator $\mathrm{O} 4$ blocks transcription as indicated. Reproduced from reference 49. transposition occurs in response to nutritional stress. ${ }^{71}$ Host DNA structuring proteins have been reported to be involved in assembly of the transpososome and in target selection. ${ }^{72-74}$ The exact roles of these DNA recombination and repair proteins in transposition have not been elucidated. 71

In some cases, the DNA adenine methylase (Dam) affects transposition, probably due to its effect on transposase transcription and activity. ${ }^{75,76}$ Similar observations have been made in yeast, in which multiple host factors are involved in transposition of retrotransposons such as Tyl and Ty3.77,78 The E. coli RpoS stationary phase sigma factor has also been shown to influence transposition rates, ${ }^{79,80}$ and unpublished observations). It is not surprising that these host proteins affect the overall transposition rates for transposons in E. coli. ${ }^{33-35,69-80}$

The mechanism recently identified for PglpFK activation provides relief from starvation and therefore could have been selected for through evolutionary time. Our results suggest that the DNA loop directly contacts RNA polymerase as the means for transcriptional activation (Figure 6). It appears to be a genuine example of directed mutation, since mutation is directed to a specific operon and occurs at a greater rate under conditions that allow benefit to the organism (i.e., in the presence of glycerol and the absence of glucose). The fact that mutation rate is influenced by the presence of glycerol in a process mediated by the glycerol repressor provides a mechanistic explanation for IS5-mediated directed mutational control. It leads us to propose that GlpR has two functions, one in controlling $g l p$ regulon gene expression as recognized previously, and the other in controlling the conformational state of the upstream DNA so as to influence the rate of IS5 insertion. These mechanisms, illustrated in Figure 7, may provide a partial explanation for the presence of four GlpR binding sites in the control region of the $g l p F K$ operon. To what extent these novel transposon-mediated adaptive evolutionary processes will prove to be applicable to other situations and organisms, poses intriguing questions for further study.

Odegard and Schatz ${ }^{81}$ have reviewed evidence for a $10^{6}$ fold increase in mutation rate in the human immune system, relative to spontaneous rates of somatic cell mutation, a phenomenon termed Somatic Hypermutation (SHM). SHM occurs in the variable regions of immunoglobulin (IgG) genes with a rate of up to $10^{-3}$ mutations/bp/cell division. In one case, point mutations arise in a specific tetranucleotide hot spot where primary sequence plays an essential role. ${ }^{81}$ While our studies have focused on mutations occurring by transposon (IS5) insertion in E. coli, the IgG system in animals involves the introduction of point mutations. Reversibility is likely to be more important in the bacterial system than in the animal system; furthermore, it is only in the bacterial system that reversibility has been demonstrated.63 Interestingly, both processes are dependent on a specific tetranucleotide hot spot sequence.

The enzyme promoting the introduction of point mutations in human IgG genes is the activation-induced cytidine deaminase. 81 By contrast, the enzyme that recognizes the tetranucleotide hot spot, CTAA in E. coli is known to be the Ins5A transposase. ${ }^{49}$ In neither case does the enzyme know what is needed. However, in the latter case, the downstream binding of GlpR to its $\mathrm{O} 1$ binding site in part determines the frequency of mutation. Since the long term benefit to the organism cannot be denied, it is intuitively clear that the occurrence of directed mutation could be a previously unrecognized property of living organisms. It is presently impossible to say how widespread the phenomenon of directed mutation is likely to be.

Many switching mechanisms have been documented in microorganisms. These include flagellar phase variation in Salmonella, controlled by inversion of a DNA segment in the chromosome 82,83 and fimbrial (fim) switching in $E$. coli, based on DNA methylation and binding of the Leucine-Responsive Protein (LRP) to specific sites in the fim control region. 84,85 Over 100 genes in Neisseria species appear to be subject to switching, including the well characterized Neisserial opa and fim loci which change antigenic properties of cell surface proteins by two different mechanisms. ${ }^{86-88}$ A well-characterized eukaryotic switch mechanism involves sex determination in homothallic yeast strains. ${ }^{89-92}$ In both cases, the ability to alternate between two or more states is beneficial to the organism, and no one questions the conclusion that these mechanisms have evolved to provide survival advantages.

The same can be argued for directed mutation. Having alternatives (i.e., low versus high frequency of a mutational event, depending on physiological need) clearly would have survival value and therefore could have evolved through natural selection. We anticipate that there will be multiple mechanisms by which this occurs. The involvement of GlpR in control of the rate of IS5 hopping upstream of the 01 GlpR binding site may merely represent the first example where the mechanism of transposon-mediated directed mutation has been elucidated.

Our preliminary results with $E$. coli suggest that while the $b g l$ and $f l h D C$ operons, both activated by IS5 insertion, are not subject to directed mutation, selection for $\mathrm{Ppd}^{+}$Fuc mutations in the fuc operon of $E$. coli may be. This last system therefore provides a potential alternative system for examining and under- 
standing transposon-mediated directed mutation. Further studies will be required to identify other examples of directed mutation to define their regulatory mechanisms and estimate their importance to organismal evolution.

\section{References}

1. Luria SE, Delbruck M. Mutations of bacteria from virus sensitivity to virus resistance. Genetics 1943;28:491-511.

2. Cairns J, Overbaugh J, Miller S. The origin of mutants. Nature 1988;335:142-5.

3. Rosenberg SM. Evolving responsively: adaptive mutation. Nat Rev Genet 2001;2: 504-15.

4. Foster PL. Mechanisms of stationary phase mutation: a decade of adaptive mutation. Annu Rev Genet 1999;33:57-88.

5. Wright BE. Stress-directed adaptive mutations and evolution. Mol Microbiol 2004;52:643-50.

6. Foster PL. Nonadaptive mutations occur on the $\mathrm{F}^{\prime}$ episome during adaptive mutation conditions in Escherichia coli. J Bacteriol 1997;179:1550-4.

7. Hendrickson H, Slechta ES, Bergthorsson $\mathrm{U}$, et al. Amplification-mutagenesis: evidence that "directed" adaptive mutation and general hypermutability result from growth with a selected gene amplification. Proc Natl Acad Sci U S A 2002;99:2164-9.

8. Roth JR, Kugelberg E, Reams AB, et al. Origin of mutations under selection: the adaptive mutation controversy. Annu Rev Microbiol 2006;60:477-501.

9. Cairns J, Foster PL. Adaptive reversion of a frameshift mutation in Escherichia coli. Genetics 1991;128:695-701.

10. Torkelson J, Harris RS, Lombardo MJ, et al. Genome-wide hypermutation in a subpopulation of stationary-phase cells underlies recombination-dependent adaptive mutation. ЕMBO J 1997;16:3303-11.

11. Harris RS, Longerich S, Rosenberg SM. Recombination in adaptive mutation. Science 1994;264:258-60.

12. Andersson DI, Slechta ES, Roth JR. Evidence that gene amplification underlies adaptive mutability of the bacterial lac operon. Science 1998;282:1133-5.

13. Ponder RG, Fonville NC, Rosenberg SM. A switch from high-fidelity to error-prone DNA double-strand break repair underlies stress-induced mutation. Mol Cell 2005; 19:791-804.

14. Layton JC, Foster PL. Error-prone DNA polymerase IV is controlled by the stressresponse sigma factor, $\mathrm{RpoS}$, in Escherichia coli. Mol Microbiol 2003;50: $549-61$.
15. Lombardo MJ, Aponyi I, Rosenberg SM. General stress response regulator RpoS in adaptive mutation and amplification in Escherichia coli. Genetics 2004;166:66980.

16. Hastings PJ, Slack A, Petrosino JF, Rosenberg SM. Adaptive amplification and point mutation are independent mechanisms: evidence for various stressinducible mutation mechanisms. PLoS Biol 2004;2:e399.

17. Slack A, Thornton PC, Magner DB, et al. On the mechanism of gene amplification induced under stress in Escherichia coli. PLoS Genet 2006;2:e48.

18. Wright BE, Minnick MF. Reversion rates in a leuB auxotroph of Escherichia coli K-12 correlate with ppGpp levels during exponential growth. Microbiol 1997;143:847-54.

19. Reimers JM, Schmidt KH, Longacre A, et al. Increased transcription rates correlate with increased reversion rates in leuB and $\operatorname{argH}$ Escherichia coli auxotrophs. Microbiol 2004;150:1457-66.

20. Wright BE, Longacre A, Reimers JM. Hypermutation in derepressed operons of Escherichia coli K12. Proc Natl Acad Sci U S A 1999;96:5089-94.

21. Cashel M, Gentry DR, Hernandez VD, Vinella D. The stringent response. In: Neidhardt FC, et al., editors. Escherichia coli and Salmonella typhimurium: Cellular and Molecular Biology. Washington, DC: ASM Press; 1996. pp. 1458-96.

22. Roth JR, Andersson DI. Amplificationmutagenesis--how growth under selection contributes to the origin of genetic diversity and explains the phenomenon of adaptive mutation. Res Microbiol 2004;155:34251.

23. Denamur E, Matic I. Evolution of mutation rates in bacteria. Mol Microbiol 2006;60: 820-7.

24. Foster PL. Stress responses and genetic variation in bacteria. Mutat Res 2005;569: 3-11.

25. Ahmed A. Evidence for replicative transposition of Tn5 and Tn9. J Mol Biol 1986;191: 75-84.

26. Dale JW. Movable genes. In: Dale JW, editor. Molecular Genetics of Bacteria. England: John Wiley \& Sons; 1998. pp. 187210.

27. Weinert TA, Schaus NA, Grindley ND. Insertion sequence duplication in transpositional recombination. Science 1983;222: 755-65.

28. Hallet B, Sherratt DJ. Transposition and site-specific recombination: adapting DNA cut-and-paste mechanisms to a variety of genetic rearrangements. FEMS Microbiol Rev 1997;21:157-78.

29. Craig NL. Mobile DNA II. Washington, DC: ASM Press; 2002.
30. Louarn JM, Bouche JP, Legendre F, Louarn J, Patte J. Characterization and properties of very large inversions of the E. coli chromosome along the origin-to-terminus axis. Mol Gen Genet 1985;201:467-76.

31. Reif HJ, Saedler H. IS1 is involved in deletion formation in the gal region of E. coli K12. Mol Gen Genet 1975;137:17-28.

32. Schneider D, Duperchy E, Coursange E, Lenski RE, Blot M. Long-term experimental evolution in Escherichia coli. IX. Characterization of insertion sequencemediated mutations and rearrangements. Genetics 2000;156:477-88.

33. Chandler M, Mahillon J. Insertion sequences revised. In: Craig NL, Craig R, Gellert M, Lambowitz AM, editors. Mobile DNA II. Washington, DC: ASM Press; 2002. pp. 305-66.

34. Galas DJ. Bacterial insertion sequences. In: Berg D, Howe M, editors. Mobile DNA. Washington, DC: American Society for Microbiology; 1989. pp. 109-62.

35. Mahillon J, Chandler M. Insertion sequences. Microbiol Mol Biol Rev. 1998;62:725-74.

36. McCalla DR. Mechanism of action of antibacterial agents. Hahn FE, editor. Berlin, Germany: Springer-Verlag; 1979.

37. Whiteway J, Koziarz P, Veall J, et al. Oxygen-insensitive nitroreductases: analysis of the roles of nfsA and nfsB in development of resistance to 5-nitrofuran derivatives in Escherichia coli. J Bacteriol 1998;180:5529-39.

38. Reynolds AE, Felton J, Wright A. Insertion of DNA activates the cryptic bgl operon in E. coli K12. Nature 1981;293:625-9.

39. Schnetz K, Rak B. IS5: a mobile enhancer of transcription in Escherichia coli. Proc Natl Acad Sci U S A 1992;89:1244-8.

40. Petersen C, Moller LB, Valentin-Hansen P. The cryptic adenine deaminase gene of Escherichia coli. Silencing by the nucleoid-associated DNA-binding protein, H-NS, and activation by insertion elements. J Biol Chem 2002;277:31373-80.

41. Chen YM, Lu Z, Lin EC. Constitutive activation of the fucAO operon and silencing of the divergently transcribed fucPIK operon by an IS5 element in Escherichia coli mutants selected for growth on L-1,2propanediol. J Bacteriol 1989;171:6097105.

42. Barker CS, Pruss BM, Matsumura P. Increased motility of Escherichia coli by insertion sequence element integration into the regulatory region of the flhD operon. J Bacteriol 2004;186:7529-37.

43. Lin EC. Glycerol dissimilation and its regulation in bacteria. Annu Rev Microbiol 1976;30:535-78.

44. Lin ECC. Dissimilatory pathways for sugars, polyols, and carboxylates. In: 
Neidhardt F, Ingraham J, Low K, Magasanik B, Schaechter M, Umbarger H, editors. Escherichia coli and Salmonella: Cellular and Molecular Biology. 1996. pp. 307-42.

45. Zeng G, Ye S, Larson TJ. Repressor for the sn-glycerol 3-phosphate regulon of Escherichia coli K-12: primary structure and identification of the DNA-binding domain. J Bacteriol 1996;178:7080-9.

46. Weissenborn DL, Wittekindt N, Larson TJ. Structure and regulation of the glpFK operon encoding glycerol diffusion facilitator and glycerol kinase of Escherichia coli K12. J Biol Chem 1992;267:6122-31.

47. Zhang Z, Saier MH, Jr. A novel mechanism of transposon-mediated gene activation PLoS Genet,2009;5:e1000689.

48. Muramatsu S, Kato M, Kohara Y, Mizuno T. Insertion sequence IS5 contains a sharply curved DNA structure at its terminus. Mol Gen Genet 1988;214:433-8.

49. Zhang Z, Saier MH, Jr. A mechanism of transposon-mediated directed mutation. Mol Microbiol 2009;74:29-43.

50. Petes TD, Hill CW. Recombination between repeated genes in microorganisms. Annu Rev Genet 1988;22:147-68.

51. Roth JR, Benson N, Galitski T, et al. Rearrangements of the bacterial chromosome: formation and application. In: Neidhardt F, Ingraham J, Low K, Magasanik B, Schaechter M, Umbarger H, editors. Escherichia coli and Salmonella typhimurium: Cellular and Molecular Biology. Washington, DC: ASM Press; 1996. pp. 2256-76.

52. Ababneh AM, Ababneh ZQ, Large CC. DNA A-tracts bending: polarization effects on electrostatic interactions across their minor groove. J Theor Biol 2008;252:742-9.

53. Tchernaenko V, Halvorson HR, Lutter LC. Topological measurement of an A-tract bend angle: variation of duplex winding. $\mathrm{J}$ Mol Biol 2003;326:751-60.

54. Tchernaenko V, Halvorson HR, Lutter LC. Topological measurement of an A-tract bend angle: effect of magnesium. $\mathrm{J}$ Mol Biol 2004;341:55-63.

55. Saier MH, Jr. The bacterial chromosome. Crit Rev Biochem Mol Biol 2008;43:89-134.

56. Berg DE. Insertion and excision of the transposable kanamycin resisrance determinant Tn5. In: Bukhari AI, Shapiro JA, Adhya SL, editors. DNA insertion element, plasmids and episomes. Cold Spring Harbor, NY: Cold Spring harbor Laboratory; 1977. pp. 205-12.

57. Botstein D, Kleckner N. Translocation and illegitimate recombination by the tetracycline resistance element Tn10. In: Bukhari AI, Shapiro JA, Adhya SL, editors. DNA insertion elements, plasmids and episomes. Cold Spring Harbor, NY: Cold Spring Harbor Laboratory; 1977. pp. 185-
204.

58. Khatoon H, Bukhari AI. DNA rearrangements associated with reversion of bacteriophage $\mathrm{Mu}$-induced mutations. Genetics 1981;98:1-24.

59. Strauch E, Beutin L. Imprecise excision of insertion element IS5 from the fliC gene contributes to flagellar diversity in Escherichia coli. FEMS Microbiol Lett 2006;256:195-202.

60. Chakrabarti T, Chen YM, Lin EC. Clustering of genes for L-fucose dissimilation by Escherichia coli. J Bacteriol 1984;157:984-6.

61. Chen YM, Chakrabarti T, Lin EC. Constitutive activation of L-fucose genes by an unlinked mutation in Escherichia coli. J Bacteriol 1984;159:725-9.

62. Podolny V, Lin EC, Hochschild A. A cyclic AMP receptor protein mutant that constitutively activates an Escherichia coli promoter disrupted by an IS5 insertion. J Bacteriol 1999;181:7457-63.

63. Zhang Z, Yen MR, Saier MH, Jr. Precise excision of IS5 from the intergenic region between the fucPIK and the fucAO operons and mutational control of fucPIK operon expression in Escherichia coli. J Bacteriol 2010;192:2013-9.

64. Massey RC, Buckling A. Environmental regulation of mutation rates at specific sites. Trends Microbiol 2002;10:580-4.

65. Galhardo RS, Hastings PJ, Rosenberg SM. Mutation as a stress response and the regulation of evolvability. Crit Rev Biochem Mol Biol 2007;42:399-435.

66. Hastings PJ, Bull HJ, Klump JR, Rosenberg SM. Adaptive amplification: an inducible chromosomal instability mechanism. Cell 2000;103:723-31.

67. Hall BG. Activation of the bgl operon by adaptive mutation. Mol Biol Evol 1998; 15:1-5.

68. Hall BG. Spectra of spontaneous growthdependent and adaptive mutations at ebgR. J Bacteriol 1999;181:1149-55.

69. Chaconas G, Lavoie BD, Watson MA. DNA transposition: jumping gene machine, some assembly required. Curr Biol 1996; 6:817-20.

70. Swingle B, O'Carroll M, Haniford D, Derbyshire KM. The effect of host-encoded nucleoid proteins on transposition: H-NS influences targeting of both IS903 and Tn10. Mol Microbiol 2004;52:1055-67.

71. Twiss E, Coros AM, Tavakoli NP, Derbyshire KM. Transposition is modulated by a diverse set of host factors in Escherichia coli and is stimulated by nutritional stress. Mol Microbiol 2005;57: 1593-607.

72. Chalmers R, Guhathakurta A, Benjamin H, Kleckner N. IHF modulation of Tn10 transposition: sensory transduction of supercoiling status via a proposed protein/DNA molecular spring. Cell 1998;29;93:897-908.

73. Lavoie BD, Chaconas G. Site-specific HU binding in the Mu transpososome: conversion of a sequence-independent DNAbinding protein into a chemical nuclease. Genes Dev 1993;7:2510-9.

74. Surette MG, Chaconas G. A protein factor which reduces the negative supercoiling requirement in the Mu DNA strand transfer reaction is Escherichia coli integration host factor. J Biol Chem 1989;264:3028-34.

75. Yin JC, Krebs MP, Reznikoff WS. Effect of Dam methylation on Tn5 transposition. J Mol Biol 1988;199:35-45.

76. Roberts D, Hoopes BC, McClure WR, Kleckner N. IS10 transposition is regulated by DNA adenine methylation. Cell 1985;43:117-30.

77. Aye M, Irwin B, Beliakova-Bethell N, et al. Host factors that affect Ty3 retrotransposition in Saccharomyces cerevisiae. Genetics 2004;168:1159-76.

78. Scholes DT, Banerjee M, Bowen B, Curcio MJ. Multiple regulators of Tyl transposition in Saccharomyces cerevisiae have conserved roles in genome maintenance. Genetics 2001;159:1449-65.

79. Ilves H, Horak R, Kivisaar M. Involvement of sigma(S) in starvation-induced transposition of Pseudomonas putida transposon Tn4652. J Bacteriol 2001;183:5445-8.

80. Moorthy S, Mahadevan S. Differential spectrum of mutations that activate the Escherichia coli bgl operon in an rpoS genetic background. J Bacteriol 2002;184: 4033-8.

81. Odegard VH, Schatz DG. Targeting of somatic hypermutation. Nat Rev Immunol 2006;6:573-83.

82. Aldridge PD, Wu C, Gnerer J, et al. Regulatory protein that inhibits both synthesis and use of the target protein controls flagellar phase variation in Salmonella enterica. Proc Natl Acad Sci U S A 2006;103:11340-5.

83. Ikeda JS, Schmitt CK, Darnell SC, et al. Flagellar phase variation of Salmonella enterica serovar Typhimurium contributes to virulence in the murine typhoid infection model but does not influence Salmonella-induced enteropathogenesis. Infect Immun 2001;69:3021-30.

84. Casadesus J, Low D. Epigenetic gene regulation in the bacterial world. Microbiol Mol Biol Rev 2006;70:830-56.

85. Holden NJ, Uhlin BE, Gally DL. PapB paralogues and their effect on the phase variation of type 1 fimbriae in Escherichia coli. Mol Microbiol 2001;42:319-30.

86. Snyder LA, Butcher SA, Saunders NJ. Comparative whole-genome analyses reveal over 100 putative phase-variable genes in the pathogenic Neisseria spp. Microbiol 2001;147:2321-32.

87. Jordan PW, Snyder LA, Saunders NJ. 
Strain-specific differences in Neisseria gonorrhoeae associated with the phase variable gene repertoire. BMC Microbiol 2005;5:21.

88. Hill SA, Davies JK. Pilin gene variation in Neisseria gonorrhoeae: reassessing the old paradigms. FEMS Microbiol Rev 2009;33:521-30.
89. Klar AJ. The yeast mating-type switching mechanism: a memoir. Genetics 2010;186: 443-9.

90. Klar AJ, Bonaduce MJ. The mechanism of fission yeast mating-type interconversion: evidence for two types of epigenetically inherited chromosomal imprinted events. Cold Spring Harb Symp Quant Biol
1993;58:457-65.

91. Haber JE, Rogers DT, McCusker JH. Homothallic conversions of yeast matingtype genes occur by intrachromosomal recombination. Cell 1980;22:277-89.

92. Haber JE. A locus control region regulates yeast recombination. Trends Genet 1998; 14:317-21. 